\title{
Period function for Perturbed Isochronous Centres
}

\author{
Emilio Freire* \\ E. S. Ingenieros, Universidad de Sevilla, Camino de los Descubrimientos s.n. \\ 41092 Sevilla, Spain. \\ E-mail: emilio@matinc.us.es \\ Armengol Gasull ${ }^{\dagger}$ \\ Dept. de Matemàtiques, Edifici Cc, Universitat Autònoma de Barcelona. 08193 \\ Bellaterra, Barcelona, Spain. \\ E-mail: gasull@mat.uab.es \\ and \\ Antoni Guillamon $\ddagger$ \\ Dept. de Matemàtica Aplicada I, Universitat Politècnica de Catalunya. Dr. \\ Marañón $n$ 44-50. 08028, Barcelona. \\ E-mail: toni@ma1.upc.es
}

\begin{abstract}
The problems related to the Poincaré map often exhibit a similar formulation in terms of the time (or period) function associated to a continuum of periodic orbits. In this paper, parallel to the Melnikov method used to study the periodic orbits that persist after a perturbation of a centre, we present an intrinsic general formula for the derivative of the period function. This formula is obtained by exploiting the symmetries of a planar vector field $X$ having an isochronous centre, and it is applied to estimate the number of critical periods of a "close" vector field $X_{\epsilon}=X+\epsilon Y$ having a centre.
\end{abstract}

Key Words: Isochronous centres, perturbations, critical periods.

\footnotetext{
* Partially supported by the DGICYT grant number PB98-1152 and Junta de Andalucía grant number TIC-0130.

† Partially supported by the DGICYT grant number PB96-1153 and CONACIT grant number 1999SGR 00349.

$\ddagger$ Partially supported by the DGICYT grant number PB96-1153, CONACIT grant number 1999SGR 00349 and MECD grant number PR2000-0292 0046670968.
} 


\section{INTRODUCTION}

Consider a planar vector field $X$ defined in some region $\mathcal{D}$. Given a transversal section $\Sigma$ to $X$ for which one has a well defined Poincaré map $P$ in $\mathcal{D}$, one can formulate questions like: (1) is the Poincare map the identity (that is, does $X$ have a continuum of periodic orbits?); if not, (2) how many fixed points does $P$ have? (that is, how many limit cycles does $X$ have in $\mathcal{D}$ ?); (3) what is the series expansion of $P$ ? (in the case that the transversal section starts from a weak focus, what is the value of the Liapunov constants?); (4) how many fixed points of $P_{\epsilon}$ can we obtain for a neighbouring vector field $X+\epsilon Y$ ? When $X$ is a polynomial Hamiltonian vector field, $Y$ is polynomial and the study is restricted to a compact region full of periodic orbits of $X$, this last problem is usually called weakened 16th Hilbert problem.

Suppose that we have a critical point which is a centre in $\mathcal{D}$. Each point of $\Sigma$ is representative of one of the closed orbits and so, the time spent by each orbit to go back to the initial state can be defined as a function $T: \Sigma \longrightarrow \mathbb{R}$, usually called the period function. Questions like (1) is the period function a constant function (that is, does $X$ have isochronicity?); if not, (2) how many zeroes does $T^{\prime}$ have? (that is, how many critical periods does $X$ have in $\mathcal{D}$ ?); (3) what is the series expansion of $T^{\prime}$ ? (in the case that the transversal section starts from a centre, what is the value of the period constants?), are comparable to those given above for the Poincaré map and have been posed and solved for a number of families of planar vector fields.

In this paper, we treat a question similar to problem (4) described above: Consider $X$ having an isochronous centre in $\mathcal{D}$. How many zeros of $T_{\epsilon}^{\prime}$ can we obtain for a neighbouring vector field $X_{\epsilon}=X+\epsilon Y$ in a compact subset of $\mathcal{D}$ filled of periodic orbits? Notice that this problem is different to the one concerned about local critical periods generated from a suitable choice of the period constants.

In order to state our main result we first introduce some notation and recall some known characterizations of isochronous centres.

Let $X$ be a smooth vector field on the plane having an isochronous centre of period $T_{0}$ at $p \in \mathcal{D}$. It is well known that $p$ has to be a critical point such that the eigenvalues of $D X(p)$ are purely imaginary, see [2]. Consider a transversal smooth vector field to $X$ in $\mathcal{D} \backslash\{p\}, U$. For $q \in \mathcal{D}$, denote by $\varphi(t ; q)$ (respectively $\psi(h ; q)$ ) the trajectory of $X$ (respectively $U$ ) such that when $t=0$ (respectively $h=0$ ) passes trough $q$. Along the paper, when we do not care about $q$ we just denote the trajectories as $\varphi(t)$ or $\psi(h)$.

The following characterizations of isochronous centres are known: A centre point $p \in \mathcal{D}$ is an isochronous centre if and only if one of the following assertions hold: 
(i) There exists a smooth change of coordinates in a neighbourhood of $p$ that linearizes $X$ (classical result of Poincaré).

(ii) There exists a transversal vector field $U$, commuting with $X$, i.e. $[\mathrm{X}, \mathrm{U}]=0$, see $[8,9]$.

(iii) There exists a transversal vector field $U$ and a scalar function $\mu$ such that $[X, U]=\mu X$ and

$$
\int_{0}^{T_{\gamma}} \mu(x(t), y(t)) d t=0
$$

where $\gamma=\left\{\varphi(t)=(x(t), y(t)), t \in\left[0, T_{\gamma}\right]\right\}$ is any periodic orbit of $X$ in $\mathcal{D}$, and $T_{\gamma}$ is its period, see [5]. In this case, we will say that $U$ is a normalizer of $X$.

In order to give a natural framework to the problem that we study, we take $X_{\epsilon}=X+\epsilon Y$ in the variety of centres around an isochronous centre $X$. To study how many critical periods bifurcate for a small perturbation $X_{\epsilon}$, the simplest case is when a linearizing change for $X$ is known. In this case, the problem can be handled by performing the same change to $X_{\epsilon}$ and thus obtaining $\tilde{X}=-y \frac{\partial}{\partial x}+x \frac{\partial}{\partial y}$ and a new $\tilde{Y}$. Usually, the expression of $\tilde{Y}$ is much more complicated that the one of the initial $Y$. For instance, if $Y$ is a polynomial vector field in most cases $\tilde{Y}$ is no more polynomial. The study of the period function for $-y \frac{\partial}{\partial x}+x \frac{\partial}{\partial y}+\epsilon \tilde{Y}$ is a classical tool. It can be done by writing the system in polar coordinates and studying the time trough the angular equation, see for instance $[1,3,4]$ or [6, Ch. IX].

On the other hand, if we know an isochronous system and one of its commutators, see the characterization (ii) above, we will show that it is possible to detect the critical periods of $X_{\epsilon}$ by means of an intrinsic formula from a commutator of $X$. In fact, this is the main result of this paper, see the next theorem. Before stating it, we want to comment that from a linearizing change of coordinates it is easy to get a commutator of $X$ (it is just the vector field obtained by applying the same change of variables to $x \frac{\partial}{\partial x}+y \frac{\partial}{\partial y}$ ), but on the other way around, from a commutator $U$ we do not know how to construct the linearizing change.

Theorem 1. Suppose that a vector field $X$ on the plane has an isochronous centre of period $T_{0}$ in $\mathcal{D}$. Consider a transversal vector field $U$ such that $[X, U]=0$. Let $\gamma(h):=\left\{\varphi(t ; \psi(h)), t \in\left[0, T_{0}\right]\right\}$ be the set of periodic orbits of $X$ in $\mathcal{D}$ parameterized by the time flow of $U$. Consider the family of vector fields $X_{\epsilon}=X+\epsilon Y$ having also a centre; write $Y$ as $Y=a X+b U$ and denote by $\gamma_{\epsilon}(h)$ a generic closed orbit of $X_{\epsilon}$ passing through $\psi(h)$.

Then, the following assertions hold: 
(i) The period function associated to $\gamma_{\epsilon}(h)$ is

$$
T_{\gamma_{\epsilon}}(h)=T_{0}+\epsilon T_{1}(h)+\mathcal{O}\left(\epsilon^{2}\right)
$$

where

$$
T_{1}(h)=-\int_{0}^{T_{0}} a(\varphi(t ; \psi(h))) d t
$$

(ii) The derivative of $T_{1}$ with respect to $h$ is:

$$
T_{1}^{\prime}(h)=-\left.\int_{0}^{T_{0}} \nabla a(x) \cdot U(x)\right|_{x=\varphi(t ; \psi(h))} d t .
$$

(iii) If $h^{*}$ is an simple zero of $T_{1}^{\prime}(h)$ then for small $\epsilon$ there is exactly one critical period of $X_{\epsilon}$ close to $h^{*}$ which tends to $h^{*}$ as $\epsilon$ tends to zero.

In Section 2 we give some preliminary results and prove Theorem 1. Finally, in Section 3 we give two examples of application: potential systems and Loud's systems.

\section{PROOF OF THE MAIN RESULTS}

To prove Theorem 1 we first need some auxiliary results. Suppose that we know a transversal normalizer $U$ for a particular vector field $X$, that is $[X, U]=\mu X$ for some scalar function $\mu$. We define the scalar function $V=U^{\perp} \cdot X$, where $(a, b)^{\perp}=(-b, a)$, and the dot denotes the usual scalar product. We list some of their features in the following lemma. To fix notation, we recall that, as usual, given a $\mathcal{C}^{1}$ function $f: \mathbb{R}^{2} \rightarrow \mathbb{R}^{2}$ and a $\mathcal{C}^{1}$ planar closed curve $\gamma$ parameterized by $t$ and with period $T, \gamma=$ $\{\mathrm{x}(t), t \in[0, T]\}$, then $\oint_{\gamma} f=\int_{0}^{T} f(\mathrm{x}(t)) \cdot \mathrm{x}^{\prime}(t) d t$.

LEMMA 2. Let $X$ and $U$ be two smooth transversal vector fields and $\mu$ and $V$ two scalar functions such that $[X, U]=\mu X$ and $V=U^{\perp} \cdot X$. Let $\gamma=\gamma(t)$ be a periodic solution of $X$, and $T$ its period. Then, in the domains where the involved functions are well-defined, the following properties hold:
(a) $\nabla V \cdot X=(\operatorname{div} X) V$,
(b) $\operatorname{div} \frac{X}{V}=0$
(c) $\oint_{\gamma}(\operatorname{div} X) U^{\perp}=\oint_{\gamma} \nabla V=0$,
(d) $\mu=\operatorname{div} U-\frac{\nabla V \cdot U}{V}$,
(e) $\operatorname{div} \frac{U}{V}=\frac{\mu}{V}$,
(f) $T=\oint_{\gamma} \frac{U^{\perp}}{V}$. 
Proof. Straightforward computations give that $\nabla V \cdot X-(\operatorname{div} X) V=$ $[X, U] \cdot X^{\perp}$. Then, using that $[X, U]=\mu X$, we have

$$
\nabla V \cdot X-(\operatorname{div} X) V=\mu X \cdot X^{\perp}=0,
$$

and so statement (a) is proven.

Using the formula $\operatorname{div}\left(\frac{1}{V} X\right)=\frac{1}{V^{2}}((\operatorname{div} X) V-\nabla V \cdot X)$, statement (b) follows from (a).

Again by (a), we see that

$$
\begin{aligned}
\oint_{\gamma}(\operatorname{div} X) U^{\perp} & =\left.\int_{0}^{T}(\operatorname{div} X) U^{\perp} \cdot X\right|_{\gamma(t)} d t= \\
& \left.\int_{0}^{T} \nabla V \cdot X\right|_{\gamma(t)} d t=\oint_{\gamma} \nabla V=0,
\end{aligned}
$$

which proves (c).

We use now two expressions of $\left[\frac{1}{V} X, U\right]$ :

1. If $U^{\perp} \cdot W=1$ it can be checked that the Lie bracket between both vector fields writes as $[W, U]=(\operatorname{div} U) W-(\operatorname{div} W) U$. By applying this last equality when $W=\frac{1}{V} X$ since, by (b) $\operatorname{div} W=0$, we get $\left[\frac{1}{V} X, U\right]=$ $\frac{\operatorname{div} U}{V} X$

2. On the other hand, expanding the Lie bracket by its definition, we obtain that

$$
\begin{aligned}
{\left[\frac{1}{V} X, U\right] } & =D U\left(\frac{1}{V} X\right)-\left(\frac{1}{V} D X-\frac{X(\nabla V)^{t}}{V^{2}}\right) U=\frac{1}{V}[X, U]+\frac{X(\nabla V)^{t}}{V^{2}} U \\
& =\mu \frac{1}{V} X+\frac{\nabla V \cdot U}{V^{2}} X=\left(\mu+\frac{\nabla V \cdot U}{V}\right) \frac{1}{V} X
\end{aligned}
$$

where $(\nabla V)^{t}$ denotes the transposed vector and hence $X(\nabla V)^{t}$ is a $2 \times 2$ matrix. Comparing them, we get $(d)$.

As in (b), we have that $\operatorname{div}\left(\frac{1}{V} U\right)=\frac{1}{V^{2}}((\operatorname{div} U) V-\nabla V \cdot U)$. Using (d), we prove (e):

$$
\operatorname{div}\left(\frac{1}{V} U\right)=\frac{1}{V^{2}}\left(\left(\mu+\frac{\nabla V \cdot U}{V}\right) V-\nabla V \cdot U\right)=\frac{\mu}{V}
$$

Finally, (f) follows using the definition of $V$ from

$$
\oint_{\gamma} \frac{U^{\perp}}{V}=\left.\int_{0}^{T} \frac{U^{\perp}}{V} \cdot X\right|_{\gamma(t)} d t=\int_{0}^{T} d t=T .
$$


Remark 3. From last lemma, one can also deduce that $\mu=0$ is a sufficient condition of isochronicity, as stated in (ii) in the Introduction. By the Stokes theorem, subtracting two periods of the same period annulus corresponding to $\gamma_{1}$ and $\gamma_{2}$, and using statement (e) we have:

$$
T_{\gamma_{2}}-T_{\gamma_{1}}=\oint_{\gamma_{2}} \frac{U^{\perp}}{V}-\oint_{\gamma_{1}} \frac{U^{\perp}}{V}=\iint_{I n t\left(\gamma_{2} \backslash \gamma_{1}\right)} \frac{\mu}{V}=0
$$

\subsection{Proof of Theorem 1}

Recall that $X$ is a vector field having an isochronous centre with a commutator $U$ and that $X_{\epsilon}=X+\epsilon Y$ is a perturbation of $X$ having a centre. In particular, since each vector field with a non degenerated centre possesses a symmetry group, we know that $X_{\epsilon}$ has a normalizer $U_{\epsilon}$. We write it as $U_{\epsilon}=U+\epsilon W+\mathcal{O}\left(\epsilon^{2}\right)$. In the spirit of Lemma 2, we compute

$$
V_{\epsilon}=U_{\epsilon}^{\perp} \cdot X_{\epsilon}=U^{\perp} \cdot X+\epsilon\left(U^{\perp} \cdot Y+W^{\perp} \cdot X\right)+\mathcal{O}\left(\epsilon^{2}\right) .
$$

By Lemma 2.(f), $T_{\gamma_{\epsilon}}=\oint_{\gamma_{\epsilon}} \frac{U_{\epsilon}^{\perp}}{V_{\epsilon}}$. Then, defining $\tilde{V}=U^{\perp} \cdot Y+W^{\perp} \cdot X$ and expanding $\frac{U_{\epsilon}^{\perp}}{V_{\epsilon}}$, we have:

$$
T_{\gamma_{\epsilon}}=\oint_{\gamma_{\epsilon}} \frac{U_{\epsilon}^{\perp}}{V_{\epsilon}}=\oint_{\gamma_{\epsilon}} \frac{U^{\perp}}{V}+\epsilon \oint_{\gamma_{\epsilon}}\left(\frac{W^{\perp}}{V}-\frac{\tilde{V}}{V} \frac{U^{\perp}}{V}\right)+\mathcal{O}\left(\epsilon^{2}\right) .
$$

Let $D_{\epsilon}$ denote the annulus with boundaries $\gamma_{\epsilon}$ and $\gamma_{0}$. By using Stokes Theorem and Lemma 2.(e):

$$
\oint_{\gamma_{\epsilon}} \frac{U^{\perp}}{V}-\oint_{\gamma_{0}} \frac{U^{\perp}}{V}=\iint_{D_{\epsilon}} \operatorname{div} \frac{U}{V} d x d y=\iint_{D_{\epsilon}} \frac{\mu}{V} d x d y=0 .
$$

From the last formula, formula (3) and Lemma 2.(f), $T_{\gamma_{\epsilon}}=T_{0}+\epsilon T_{1}+\mathcal{O}\left(\epsilon^{2}\right)$, where $T_{0}$ is the period of the unperturbed isochronous system and

$$
T_{1}=\oint_{\gamma_{0}}\left(\frac{W^{\perp}}{V}-\frac{\tilde{V}}{V} \frac{U^{\perp}}{V}\right)
$$

We express now $Y$ and $W$ in $\{X, U\}$-coordinates as $Y=a X+b U$, $W=\alpha X+\beta U$. Obviously,

$\tilde{V}=U^{\perp} \cdot Y+W^{\perp} \cdot X=U^{\perp} \cdot(a X+b U)+\left(\alpha X^{\perp}+\beta U^{\perp}\right) \cdot X=(a+\beta) V$. 
Now, we take any point $q \in \gamma_{0}$ and consider the trajectory $\psi(h ; q)$ of $U$ such that $\psi(0 ; q)=q$. Remember that $\varphi(t ; x)$ is a trajectory of $X$.

Then, using that $\oint_{\gamma_{0}} f=\left.\int_{0}^{T_{0}} f \cdot X\right|_{\varphi(t ; q)} d t$, we get that

$$
T_{1}=\oint_{\gamma_{0}} \frac{\alpha X^{\perp}+\beta U^{\perp}}{V}-(a+\beta) \frac{U^{\perp}}{V}=-\int_{0}^{T_{0}} a(\varphi(t ; q)) d t
$$

and the proof of (i) follows.

Let us prove (ii). Now we think of $\gamma_{0}$ as a generic closed curve of the isochronous centre. Observe that fixed $X, U$, whatever $Y$ is, in $T_{1}$ we are always integrating along a solution of $X$ and the parameterization of $T_{1}$ can be given by $h$,

$$
T_{1}=T_{1}(h)=-\int_{0}^{T_{0}} a(\varphi(t ; \psi(h ; q))) d t .
$$

Remember that the commutation of flows generated by $X$ and $U$,

$$
\varphi(t ; \psi(h ; q))=\psi(h ; \varphi(t ; q))),
$$

is equivalent to $[X, U]=0$.

Then,

$$
\begin{aligned}
T_{1}^{\prime}(h) & =-\int_{0}^{T_{0}} \frac{\partial}{\partial h} a(\varphi(t ; \psi(h ; q))) d t=-\int_{0}^{T_{0}} \frac{\partial}{\partial h} a(\psi(h ; \varphi(t ; q))) d t= \\
& -\int_{0}^{T_{0}} \nabla a(\psi(h ; \varphi(t ; q))) \cdot U(\psi(h ; \varphi(t ; q))) d t= \\
& -\int_{0}^{T_{0}} \nabla a(\varphi(t ; \psi(h ; q))) \cdot U(\varphi(t ; \psi(h ; q))) d t .
\end{aligned}
$$

Finally, since

$$
\frac{\partial}{\partial h} T_{\gamma_{\epsilon}}(h)=\epsilon T_{1}^{\prime}(h)+\mathcal{O}\left(\epsilon^{2}\right),
$$

(ii) follows. The last statement of the theorem is a direct consequence of the implicit function theorem.

\section{EXAMPLES}

In this section we apply our method to two examples. The first one is the case of potential systems. The second one is a class of quadratic centres. We remark that in both cases the linearizing method would also be applicable. Anyway, as far as we know, the results that we obtain by using our method are new. 
Proposition 4. Consider the system

$$
\left\{\begin{array}{l}
\dot{x}=-y \\
\dot{y}=x+\epsilon G^{\prime}(x) .
\end{array}\right.
$$

Then, for $\epsilon$ small enough, the zeroes of

$$
I(s)=\left.\int_{0}^{2 \pi} \frac{x\left(x G^{\prime \prime}(x)-G^{\prime}(x)\right)}{x^{2}+y^{2}}\right|_{x=s \cos t, y=s \sin t} d t
$$

give rise to critical periods of (5).

Moreover, if $G^{\prime}(x)$ is a polynomial of degree $n$ then the maximum number of simple zeroes of $I$ is $n-2$. In other words, fixed any compact set, at most $n-2$ critical periods bifurcate from the closed orbits of $(5)_{\epsilon=0}$.

This number coincides with the number of local critical points for the even potentials described by Chicone and Jacobs using period constants in [1, Thm. 4.3].

Proof. In the notation of Theorem $1, X=(-y, x), U=(x, y)$ and $Y=\left(0, G^{\prime}(x)\right)$. Hence, $a=\frac{x G^{\prime}(x)}{x^{2}+y^{2}}, b=\frac{y G^{\prime}(x)}{x^{2}+y^{2}}$ and

$$
\nabla a \cdot U=\frac{x\left(x G^{\prime \prime}(x)-G^{\prime}(x)\right)}{x^{2}+y^{2}} .
$$

Taking $\psi(h)=\left(e^{h}, 0\right), \varphi(t ; \psi(h))=\left(e^{h} \cos t, e^{h} \sin t\right)$ and renaming $e^{h}$ by $s$ the proof follows by applying Theorem 1. The proof that the maximum number of simple zeros of the above expression is $n-2$ when $G^{\prime}$ is a polynomial of degree $n$ is straightforward.

In [7], Loud gave a compact description of all the isochronous systems existing in the quadratic family. That is, he showed that every quadratic system having an isochronous centre can be brought under a change of variables into a system of the form

$$
\left\{\begin{array}{l}
\dot{x}=-y+B x y \\
\dot{y}=x+D x^{2}+F y^{2}
\end{array}\right.
$$

where the pairs $(D / B, F / B) \in S$ with $S=\{(0,1),(0,1 / 4),(-1 / 2,2)$, $(-1 / 2,1 / 2)\}$. Note that for all the values of $(B, D, F)$, system $(6)$ has a reversible centre at the origin.

We will here study the period function for systems that are close to one of the above isochronous systems, namely, systems of type:

$$
\left\{\begin{array}{l}
\dot{x}=-y+(1+\epsilon \tilde{B}) x y, \\
\dot{y}=x+(0+\epsilon \tilde{D}) x^{2}+(1+\epsilon \tilde{F}) y^{2},
\end{array}\right.
$$


with $(D / B, F / B)=(0,1)$ and $\epsilon$ small enough. We prove the following result:

Proposition 5. Consider the system (7). Fix a compact set $K$ in the region filled of periodic orbits of (7) $\epsilon=0$. Then, for $\epsilon$ small enough, system (7) has at most one critical period in $K$. Furthermore, the critical period can appear only when $\frac{\tilde{B}-\tilde{F}}{\tilde{D}}>3$.

Proof. Write $X_{\epsilon}=X+\epsilon Y$, where $X$ is defined by

$$
\left\{\begin{array}{l}
\dot{x}=-y+x y \\
\dot{y}=x+y^{2}
\end{array}\right.
$$

We can easily check that if $U$ is

$$
\left\{\begin{array}{l}
\dot{x}=(1-x) x / 2 \\
\dot{y}=(1-x) y / 2
\end{array}\right.
$$

then $[X, U]=0$. Observe that $\psi(h)=\left(\frac{e^{h / 2}}{1+e^{h / 2}}, 0\right)$ is a trajectory of $U$. Also, $X=V X_{H}$, with $V(x)=(1-x)^{3} / 2$ and $H(x, y)=\frac{x^{2}+y^{2}}{(1-x)^{2}}$. Hence, the trajectory of $X$ starting at $\psi(h)$ can be written as $\varphi(t ; \psi(h))=$ $\left(x_{h}(t), y_{h}(t)\right)$, where

$$
x_{h}(t)=\frac{e^{h / 2} \cos t}{1+e^{h / 2} \cos t}, \quad y_{h}(t)=\frac{e^{h / 2} \sin t}{1+e^{h / 2} \cos t} .
$$

It also turns out that $Y=a X+b U$ with

$a=\frac{x}{x^{2}+y^{2}} \quad$ and $\quad b=\frac{2 y\left(\tilde{D} x^{2}+\tilde{F} y^{2}+\tilde{B} x^{2}+x\left(-\tilde{D} x^{2}+(\tilde{B}-\tilde{F}) y^{2}\right)\right)}{(1-x)\left(x^{2}+y^{2}\right)}$.

Following formula (2) in Theorem 1 we get that

$\left.T_{1}^{\prime}(h)=-\frac{1}{2} \int_{0}^{2 \pi}\left(1-x_{h}(t)\right) \frac{x_{h}(t)}{x_{h}(t)^{2}+y_{h}(t)^{2}}\left(\tilde{D} x_{h}(t)^{2}+(\tilde{F}-\tilde{B}) y_{h}(t)^{2}\right)\right) d t$.

To obtain a more compact expression of $T_{1}^{\prime}(h)$ we introduce, after integration, the change of parameter $z=Z(h):=\sqrt{\frac{1-e^{h / 2}}{1+e^{h / 2}}}$. Since $h$ ranges between $-\infty$ and 0 , it is clear that $z \in(0,1)$. Thus, tedious computations give 
$\left.T_{1}^{\prime}(h)\right|_{h=Z^{-1}(z)}=\pi(z-1)^{2}\left(1+z^{2}\right) \frac{\tilde{D}\left(z^{2}+4 z+1\right)\left(1+z^{2}\right)+(\tilde{F}-\tilde{B}) 4 z^{2}}{8 z^{3}\left(z^{2}+2 z+1\right)}$,

and for $z \in(0,1)$,

$$
T_{1}^{\prime}=0 \Leftrightarrow \Lambda(z):=\frac{1}{4} \frac{\left(z^{2}+4 z+1\right)\left(1+z^{2}\right)}{z^{2}}=\frac{\tilde{B}-\tilde{F}}{\tilde{D}} .
$$

It turns out that $\frac{d}{d z} \Lambda(z)=\frac{1}{2 z^{3}}(z-1)(z+1)^{3}$ is negative for all $z \in(0,1)$. Then, by using part (iii) of Theorem 1, system (7) for $\epsilon$ small enough has at most one critical period in $K$. Notice that since $\Lambda(0,1)=(3,+\infty)$, there exists a $z^{*}$ such that $\Lambda\left(z^{*}\right)=\frac{\tilde{B}-\tilde{F}}{\tilde{D}}$ just in the case when $\frac{\tilde{B}-\tilde{F}}{\tilde{D}}>3$ and in this case it is unique. To have a critical period under the hypotheses of the theorem, it must happen that $\psi\left(Z^{-1}\left(z^{*}\right)\right) \in K$.

\section{REFERENCES}

1. C. Chicone, M. Jacobs. Bifurcations of critical periods for plane vector fields, Trans. Amer. Math. Soc. 312 (1989), 433-486.

2. C.J. Christopher, J. Devlin. Isochronous centers in planar polynomial systems, SIAM J. Math. Anal. 28 (1997), 162-177.

3. J.P. Françoise. The first derivative of the period function of a plane vector field, Publicacions Matemàtiques, 41 (1997), 127-134.

4. J.P. Françoise. The successive derivatives of the period function of a plane vector field, J. Differential Equations, 146 (1998), 320-335.

5. E. Freire, A. Gasull, A. Guillamon. A characterization of isochronous centres in terms of symmetries, Preprint UAB (2000).

6. S. Lefschetz. Differential equations: geometric theory, Dover Publications, Inc., New York, 1977.

7. W.S. LOUD. Behavior of the period of solutions of certain plane autonomous systems near centers, Contributions to Differential Equations 3 (1964), 21-36.

8. M. Sabatini. Characterizing isochronous centres by Lie brackets, Diff. Eq. Dyn. Sys., 5, 91-99 (1997).

9. M. Villa RINI. Regularity properties of the period function near a centre of a planar vector field, Nonlinear Analysis T.M.A. 19 (1992), 787-803. 